\title{
Lokale Allergie in der Nase
}

\author{
Viele Patienten leiden an den klassischen Symptomen einer aller- \\ gischen Rhinitis, sind aber im Pricktest unauffällig und weisen auch \\ kein spezifisches IgE im Serum auf. Möglicherweise spielen hier lokale \\ allergologische Pathomechanismen eine Rolle.
}

n einer spanischen Studie wurden drei

Gruppen untersucht: 50 Patienten mit nicht allergischer Rhinitis, 30 Patienten mit allergischer Rhinitis aufgrund einer Sensibilisierung gegen Dermatophagoides pteronyssinus sowie 30 gesunde Kontrollprobanden. Beide Patientengruppen litten seit mindestens zwei Jahren an persistierender Rhinitis. Studienteilnehmer mit Verdacht auf andere immunologische Erkrankungen waren ausgeschlossen. Bei nicht allergischer Rhinitis durfte kein Hinweis auf eine allergische Genese vorliegen.

Bei allen Studienteilnehmern wurden aus der Nasallavage die Konzentrationen von ECP („eosinophil cationic protein"), Gesamt-IgE und spezifischem IgE gegen $D$. pteronyssinus bestimmt. Patienten und Kontrollprobanden unterzogen sich außerdem einem nasalen Provokationstest mit $D$. pteronyssinus. Schließlich wurden noch die Leukozyten aus dem Nasensekret analysiert.

In der Nasallavage wiesen sowohl Patienten mit allergischer als auch mit nicht allergischer Rhinitis erhöhte ECPWerte auf im Vergleich zu den Kontroll-

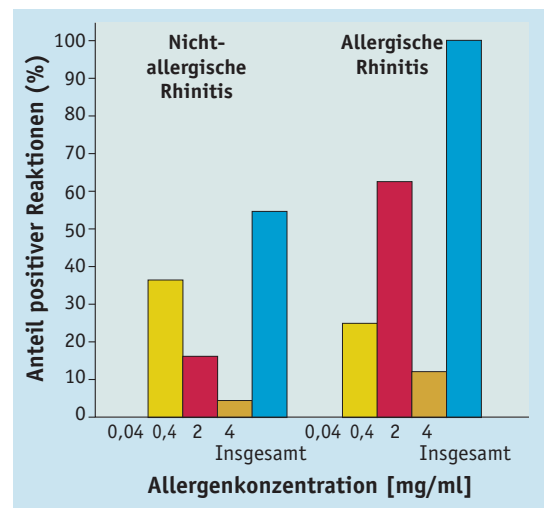

Auch bei nicht allergischer Rhinitis reagieren $\mathbf{5 4 \%}$ der Patienten im nasalen Provokationstest mit Der $\mathbf{p} 1$ positiv. probanden. Die Werte der beiden Rhinitis-Gruppen unterschieden sich dabei nicht signifikant voneinander. GesamtIgE und spezifisches IgE waren allerdings bei den Allergiekranken höher.

Im nasalen Provokationstest reagierten alle Patienten mit allergischer Rhinitis positiv. In der Gruppe der nicht allergischen Rhinitispatienten zeigten immerhin 54\% eine positive Reaktion. Eine positive Reaktion beim Provokationstest korrelierte dabei mit der Konzentration des spezifischen $\operatorname{IgE}$ und der Eosinophilenzahl im Nasensekret. Patienten mit allergischer Rhinitis und mit nicht allergischer Rhinitis wiesen in der Nasallavage vergleichbare Dichten von CD45+-Zellen, Neutrophilen, Eosinophilen, Basophilen und $\mathrm{CD}^{+}{ }^{-} / \mathrm{CD}^{+}-\mathrm{T}$-Zellen auf.

Fazit: Auch wenn bei einer persistierenden Rhinitis systemische Zeichen einer Allergie fehlen, können bei einem Teil der Patienten lokal in der Nase allergisch ausgelöste entzündliche Reaktionen beobachtet werden.

Rondón C et al. Local IgE production and positive nasal provocation test in patients with persistent nonallergic rhinitis. J Allergy Clin Immunol 2007; 119: 899-905

\section{Drei Therapiejahre sind besser als zwei}

\section{Die Langzeitwirkung der sublingualen Immuntherapie (SLIT) bei} saisonaler allergischer Rhinitis ist in klinischen Studien gezeigt worden. Die Datenlage für die SLIT bei perennialer Rhinitis ist dagegen noch inkonsistent, Langzeitstudien wurden bisher kaum publiziert.

$\mathrm{n}$ n einer doppelblinden randomisierten Langzeitstudie wurden die Wirksamkeit einer zwei- oder einer dreijährigen SLIT sowie ein möglicher Effekt über diese Zeit hinaus bei Patienten mit Hausstaubmilbenallergie untersucht. Die 137 Patienten erhielten entweder über zwei Jahre eine SLIT mit Allergenextrakten von Dermatophagoides pteronyssinus und D. farinae (ALK-Abelló) und dann über ein Jahr eine Plazebo-SLIT ( $n=67$ ) oder die aktive Behandlung über alle drei Jahre $(\mathrm{n}=70)$. Drei Jahre nach Behandlungsende wurden die Patienten dann nochmals untersucht. Als Parameter für den Therapieerfolg dienten u.a. Veränderungen in der Symptomatik und im nasalen Provokationstest.

Während der ersten drei Jahre ging der Gesamtscore, berechnet aus Symptomscore und nasalem Widerstand, in beiden Gruppen signifikant zurück. Die Ergebnisse für die Gruppe mit drei aktiven Therapiejahren waren dabei signifikant besser als die Ergebnisse der Gruppe mit nur zwei Jahren. Unterschiedlich waren auch die Ergebnisse des Follow-up drei Jahre nach Thera- pieende: Bei Patienten mit dreijähriger Behandlung war der Wiederanstieg des Gesamtscores weniger stark ausgeprägt als bei Patienten nach nur zweijähriger aktiver Therapie.

Schwerwiegende lokale oder systemische Reaktionen wurden nicht registriert, häufigste Nebenwirkungen waren oraler Pruritus, Rhinitis und gastrointestinale Störungen.

Fazit: Auch in der Behandlung der perennialen allergischen Rhintis ist die SLIT wirksam und sicher. Eine dreijährige Behandlung führt $\mathrm{zu}$ signifikant besseren Therapieergebnissen als eine nur zweijährige Therapie.

Tahamiler $\mathbf{R}$ et al. Long-term efficacy of sublingual immunotherapy in patients with perennial rhinitis. Laryngoscope 2007; 117: 965-9 